\title{
Persistent Allopeptide Reactivity and Epitope Spreading in Chronic Rejection of Organ Allografts
}

\author{
Rodica Ciubotariu, ${ }^{\star}$ Zhuoru Liu, ${ }^{\star}$ Adriana I. Colovai, ${ }^{\star}$ Eric Ho, ${ }^{\star}$ Sylviu Itescu, ${ }^{\ddagger}$ Stefano Ravalli, ${ }^{\S}$ Mark A. Hardy,,${ }^{\ddagger}$ \\ Raffaello Cortesini, $\|$ Eric A. Rose, ${ }^{\ddagger}$ and Nicole Suciu-Foca* \\ College of Physicians and Surgeons of Columbia University, *Department of Pathology, ${ }^{\ddagger}$ Department of Surgery, ${ }^{\S}$ Department of Internal \\ Medicine, New York 10032; and "Università degli Studi di Roma "La Sapienza," Instituto di II Clinica Chirurgica, Servizio Trapianti \\ d'Organo, Roma, Italy
}

\begin{abstract}
The role of the indirect allorecognition pathway in acute allograft rejection has been documented both in organ recipients and in experimental models. However, it is unknown whether self-restricted recognition of donor alloantigens also contributes to chronic allograft rejection. The aim of this study was to determine the relationship between allopeptide reactivity, epitope spreading, and chronic rejection. Using synthetic peptides corresponding to the hypervariable region of 32 HLA-DR alleles, we have followed the specificity of self-restricted $\mathrm{T}$ cell alloresponses to the donor in a population of 34 heart allograft recipients. $T$ cells from sequential samples of blood collected from the patients up to $36 \mathrm{mo}$ after transplantation were studied in limiting dilution analysis for allopeptide reactivity. The incidence of coronary artery vasculopathy (CAV) was significantly higher in patients who displayed persistent alloreactivity late after transplantation than in patients who showed no alloreactivity after the first 6 mo after transplantation. Both intra- and intermolecular spreading of epitopes was observed with an increased frequency in patients developing CAV in less than $2 \mathrm{yr}$, compared with patients without CAV; this suggests that diversification of the immune response against the graft contributes to chronic rejection. These data provide a strategy for identifying patients at risk of developing CAV and a rationale for therapeutic intervention aimed to prevent the progression of the rejection process. (J. Clin. Invest. 1998. 101:398-405.) Key words: indirect allorecognition • coronary artery vasculopathy - reactivity to HLA-DR allopeptides • intramolecular spreading • intermolecular spreading
\end{abstract}

\section{Introduction}

Coronary arteriosclerosis of the graft, a manifestation of chronic rejection, frequently limits the long-term success of cardiac transplantation (1). Despite intensive research efforts,

Address correspondence to Dr. Nicole Suciu-Foca, College of Physicians and Surgeons of Columbia University, Department of Pathology, 630 West 168th Street, PS 14-401, New York, NY 10032. Phone: 212-305-6941; FAX: 212-305-3429; E-mail: Suciu_Foca@cuccfa.ccc. columbia.edu

Received for publication 7 July 1997 and accepted in revised form 6 November 1997.

J. Clin. Invest.

(C) The American Society for Clinical Investigation, Inc. 0021-9738/98/01/0398/08 \$2.00

Volume 101, Number 2, January 1998, 398-405

http://www.jci.org the underlying causes of chronic rejection which affects most solid organ transplants such as heart, kidney, and liver remain unknown. However, since the length of transplant survival is inversely related to the number of HLA antigen mismatches carried by the graft (2-5), and since graft atherosclerosis occurs more often in patients who develop antidonor HLA antibodies after transplantation, it is suspected that this condition is driven by the host's immune response against the allogeneic MHC antigens which it recognizes (6-11).

Histologically, the most prominent features of allograft arteriosclerosis consist of perivascular inflammation and generalized concentric intimal thickening characterized by the mingling of $\mathrm{T}$ cells and macrophages among the smooth muscle cells of the intima (12-14). It has been suggested that cytokines secreted by lymphocytes, macrophages, and by cells of the inflamed tissue could be responsible for the formation of arteriosclerotic lesions. These cytokines include IL-2, IL-6, IL-10, IFN- $\gamma$, and growth factors such as platelet-derived growth factor, insulin-like growth factor, and fibroblast growth factor, which can induce or promote smooth muscle cell proliferation (15-20). Therefore, it is possible that chronic antigenic stimulation of T cells by graft HLA antigens results in the production of lymphokines which damage the vascular endothelium. The endothelial cells may then secrete growth factors which induce the proliferation of smooth muscle cells and the migration of myocytes from the media into the intima (12).

$\mathrm{T}$ cell recognition of foreign MHC antigens occurs by two mechanisms: direct allorecognition of intact donor MHC molecules expressed on the membrane of graft cells and indirect allorecognition of donor MHC molecules which are processed and presented as peptides bound to the MHC class II molecules displayed on the surface of host antigen-presenting cells $(\mathrm{APCs})^{1}(21-24)$.

Direct allorecognition is unlikely to contribute to late rejection because it is triggered primarily by passenger dendritic cells of donor origin which migrate out of the graft early after transplantation $(25,26)$. Furthermore, T cells which recognize donor alloantigens on "nonprofessional" APCs that lack costimulatory elements become anergized (27-30).

It is more likely that the indirect pathway of $\mathrm{T}$ cell activation which is stimulated by allopeptides presented by professional APCs (dendritic cells, macrophages, B cells) of host origin is the major contributor to chronic rejection $(27,31)$. Since there is a continuous supply of donor allopeptides processed and presented by host APCs, self-MHC restricted T helper (Th) cells may perpetuate the alloimmune response causing chronic rejection (21-24, 27-31).

1. Abbreviations used in this paper: APCs, antigen-presenting cells; CAV, coronary artery vasculopathy; EMB, endomyocardial biopsies; LDA, limiting dilution analysis; TCL, T cell lines; Th, T helper. 
In previous studies, we have demonstrated that acute rejection of heart transplants is strongly associated with the appearance both in the graft and in the recipient's circulation of $\mathrm{T}$ cells which react to an immunodominant determinant of a donor HLA-DR antigen. Although the occurrence of multiple acute rejection episodes were accompanied by intermolecular spreading, no intramolecular epitope spreading was found during the early posttransplantation period (31). These findings suggested that tolerance to the dominant determinant of donor HLA molecules may be induced to prevent allograft rejection, a strategy proved to be effective in the therapy of autoimmune diseases (32-34).

However, evidence has been accumulating that the $\mathrm{T}$ cell repertoire is dynamic and that intramolecular and intermolecular epitope spreading to additional specificities occurs during the course of both experimentally induced and spontaneous autoimmune diseases $(35,36)$. It is possible that epitope spreading also accounts for the chronicity of $\mathrm{T}$ cell reactivity and production of inflammatory cytokines involved in the pathogenesis of graft atherosclerosis. $T$ cells which react specifically to the immunodominant epitope may induce an inflammatory cascade in the graft, resulting in acute rejection. Tissue debris processed by macrophages and antigen specific B cells in the peripheral lymphoid tissue may amplify the inflammatory response due to the recruitment of a diversified repertoire of $\mathrm{T}$ cells reacting to subdominant and/or cryptic epitopes. To explore this hypothesis, we have investigated the indirect alloimmune response occurring late after transplantation in heart allograft recipients, followed sequentially up to 36 mo. Our results demonstrate, for the first time, that chronic rejection is characterized by persistent allopeptide reactivity and both inter-and intramolecular epitope spreading.

\section{Methods}

Patients. The study population consisted of 34 recipients of primary heart allografts ( 28 males and 6 females). 30 patients were Caucasoids and 4 were African-Americans. All gave informed consent under the auspices of the appropriate Institutional Review Board. The mean age of the patients was $53.1 \pm 8.7 \mathrm{yr}$, ranging from 29 to $65 \mathrm{yr}$. Acute rejection was monitored by endomyocardial biopsies (EMB) according to a standard time schedule as described previously (31). Blood specimens were obtained for limiting dilution analysis (LDA) testing at the time of EMB. The mean number of specimens tested was $6.7 \pm 3.1$. The follow-up period ranged from 330 to 1,090 d. Baseline angiogram was performed within 1 mo of transplant to detect unsuspected vascular disease in the donor. Angiograms were repeated at first, second, and third year anniversaries of the transplant procedure. The diagnosis of coronary artery vasculopathy (CAV) was made when contrast angiography demonstrated diffuse small vessel involvement, defined as concentric, symmetrical narrowing of terminal branches. Discrete, asymmetric narrowing of the large vessels of the coronary vasculature, in the absence of peripheral small vessel pruning, was not considered diagnostic of CAV. In the absence of quantitative measurements, e.g., using intravenous ultrasonography, our criteria will likely underestimate the frequency of CAV, identifying those cases with greatest severity and reducing the possibility of falsepositive results.

Standard triple therapy immunosuppression (cyclosporine, steroids, and either azathioprine or mycophenolate mofetil) was initiated perioperatively for all patients. Cellular rejection episodes were treated either with steroid pulses (oral or intravenous) or cytolytic therapy (OKT3 or ATGAM).
HLA typing. All recipients and donors were typed for HLA-DR antigens by molecular methodology using PCR-SSOP (sequence specific oligonucleotide probes). Allelic subtypes of the same antigen were considered mismatches. 7 patients were transplanted with a graft mismatched by one HLA-DR $\beta 1$ allele and 27 with a two-DR-mismatched graft.

Peptides. Synthetic peptides corresponding to residues 1-19, 2139 , or 62-80 of DR 11 chain from 32 HLA-DR alleles (31) were obtained from Chiron Mimotopes (San Diego, CA) and Peptide Innovation (Raleigh, NC). The purity of peptides was $>90 \%$ as indicated by HPLC and mass spectrometry.

LDA of allopeptide reactive Th cells. The frequency of allopeptide reactive Th cells in the peripheral blood was measured in LDA as described previously (31). Since Th precursors recognizing allopeptides are also present in the circulation of healthy individuals, the method used for testing the reactivity of transplant recipients resides entirely on assaying the response exhibited by "committed" $\mathrm{T}$ lymphocytes.

For this procedure, patients' PBMCs were first cultured in 96-well round bottom trays for $7 \mathrm{~d}$ at a concentration of $4 \times 10^{4}, 2 \times 10^{4}$, and $1 \times 10^{4}$ per well in RPMI 1640 medium supplemented with $10 \%$ human serum (Sigma Chemical Co., St. Louis, MO) and $50 \mathrm{U}$ of recombinant (r) IL-2/ml (Boehringer Mannheim, Indianapolis, IN). 24 replicate cultures were set up for each dilution of responding cells. On day 7 , the trays were washed three times and each culture was split into three aliquots. Irradiated autologous PBMCs $\left(5 \times 10^{4} /\right.$ well $)$ and a cocktail of peptides ( $1 \mu \mathrm{M}$ of each peptide) corresponding to each of the mismatched DR antigens were added to parallel plates. In negative control wells, only self PBMCs were added. After $48 \mathrm{~h}$, the cultures were labeled with $\left[{ }^{3} \mathrm{H}\right]$ thymidine and harvested $18 \mathrm{~h}$ later. Wells were considered positive if $\mathrm{cpm}$ in the cultures with allopeptides were at least three times higher than cpm in replicate control cultures without peptides and exceeded 2,000. The frequency was calculated as described previously $(37,38)$.

To study intramolecular spreading, patients were tested in LDA for reactivity against each component of the peptide cocktail covering the three hypervariable regions of the mismatched donor DR antigens. To obtain a sufficient number of $\mathrm{T}$ cells for the 3-d split-well blastogenesis assay, cells plated in LDA were expanded in rIL-2 for 2 wk before stimulation with the peptide. To determine DR restriction elements, PBMCs from hemiallogeneic donors, sharing with the recipient a single HLA-DR antigen, were used as APCs.

Development of allopeptide specific T cell lines (TCLs) for epitope mapping. To establish the hierarchy of determinants of the DR $\beta 1 * 1301$ molecule, which are recognized by HLA-DR $\beta 1 * 0701$ homozygous individuals, allopeptide specific TCLs were developed. Responding cells were PBMCs from a healthy blood donor (EAS) whose DR $\beta$ * genotype was DR $\beta 1 * 0701 / 0701$. PBMCs at $10^{6} / \mathrm{ml}$ were stimulated in 24-well plates (Nunc, Inc., Naperville, IL) with $10 \mu \mathrm{g} / \mathrm{ml}$ of individual synthetic peptides corresponding to residues $1-19,21-39$, or $62-80$ of DR $\beta 1 * 1301$ in RPMI 1640 medium supplemented with $10 \%$ pooled human serum, $2 \mathrm{mM}$ L-glutamine, and $50 \mathrm{mg} / \mathrm{ml}$ gentamicin (Gibco Laboratories, Grand Island, NY). 3 d after stimulation, rIL-2 was added at $10 \mathrm{U} / \mathrm{ml}$. The cultures were fed every 3-4 d with medium containing rIL-2 $(20 \mathrm{U} / \mathrm{ml})$ and expanded at $14-\mathrm{d}$ intervals by stimulation with irradiated autologous APCs and allopeptides. After three cycles of stimulation, the reactivity of the resulting TCLs to the peptide used for in vitro immunization and to soluble HLA-DR $\beta 1 * 1301$ protein was tested as described previously (37).

Purification of HLA-DR $\beta 1 * 1301$ protein. The HLA-DR $\beta 1 * 1301$ homozygous B lymphoblastoid cell line, 10W9065, was used as a source of HLA-DR molecules. DR molecules were purified by immunoaffinity chromatography using $\mathrm{mAb}$ L243, which recognizes a common structure of human HLA-DR antigens. The purity of the protein was assessed by SDS-PAGE (39).

Statistical analysis. The Kaplan-Meier product-limit curve was used to estimate the impact of $\mathrm{T}$ cell alloreactivity on the development of CAV. Differences between groups were compared by log 
Table I. Association between CAV and Th Reactivity to Donor Allopeptides

\begin{tabular}{cc}
\hline $\begin{array}{c}\text { Patients } \\
\text { with CAV }\end{array}$ & $\begin{array}{c}\text { Patients } \\
\text { without CAV }\end{array}$ \\
\hline$n=14$ & $n=20$
\end{tabular}

No. of LDA showing antidonor reactivity/total No. of LDA $46 / 80(57 \%) \quad 54 / 149(36 \%)$ $P=0.002$

rank statistics. The relationship between epitope spreading and the development of CAV was determined by the test of linear trend.

\section{Results}

Relationship between allopeptide reactivity and development of $C A V$. To explore the contribution of indirect allorecognition to chronic rejection, we have performed a prospective study of $\mathrm{T}$ cell reactivity to donor allopeptides in 34 recipients of heart allografts. Patients were monitored over a period of $1-3 \mathrm{yr}$ after transplantation for reactivity against synthetic peptides corresponding to the hypervariable region of the mismatched HLA-DR antigen of the donor. The time interval between sequential determinations was 1-12 wk. Results of LDA studies performed during the first 6 mo after transplantation on this population of patients demonstrated a strong correlation between $\mathrm{T}$ cell reactivity to donor allopeptides and acute rejection (31). LDA performed after this initial period showed that in some patients alloreactivity persisted in the absence of any histologic evidence of late acute rejection. 14 of the 34 recipients included in this study developed CAV in $<2 \mathrm{yr}$ after transplantation. The frequency of LDA showing antidonor reactivity was significantly higher in patients developing CAV than in patients without CAV (Table I). Patients who showed antidonor responses in $>50 \%$ of the LDA performed 6 mo after transplantation were considered to display persistent alloreactivity. Actuarial freedom from graft coronary artery disease at $2 \mathrm{yr}$ was $43 \%$ in patients with persistent antidonor alloreactivity after the first 6 mo after transplantation, and $78 \%$ in patients without such late responses. This suggests that persistent alloreactivity correlates with the development of CAV (Fig. 1).

Analysis of the relationship between late episodes of acute rejection, T cell reactivity to donor allopeptides, and the occurrence of CAV showed a significant association between allopeptide reactivity and CAV both in patients with $(n=12)$ and without $(n=11)$ late episodes of acute rejection (Fig. 2$)$. This indicates that $\mathrm{T}$ cells recognizing donor HLA-DR antigens via the indirect pathway may play an important role in the pathogenesis of graft vascular disease. Since alloreactivity was predictive of chronic rejection even in patients with no histologic evidence of late episodes of acute rejection, LDA monitoring is a valuable approach for identifying patients at risk of developing CAV. Only two patients who showed late acute rejection episodes and CAV displayed no reactivity against donor allopeptides after 6 mo. However, these two patients exhibited allopeptide reactivity in conjunction with early acute rejection episodes (31).

Intra- and intermolecular epitope spreading in chronic rejection. Studies of autoimmune diseases have suggested that

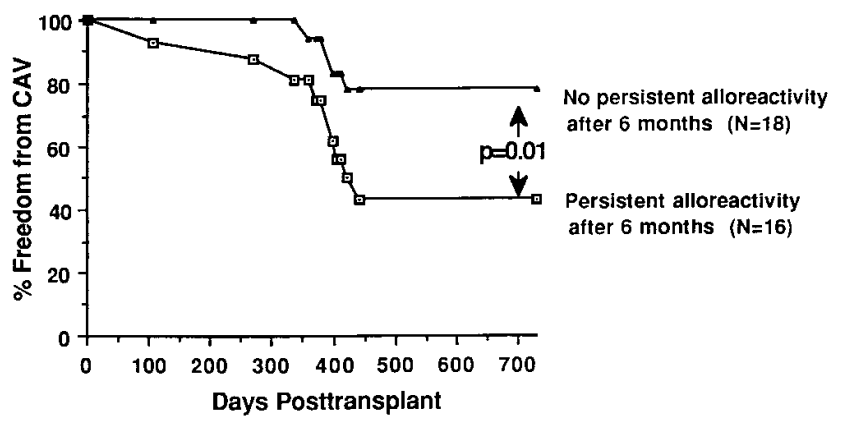

Figure 1. Actuarial freedom from CAV in heart transplant recipients with persistent reactivity to donor allopeptides. Serial blood samples were obtained from heart allograft recipients and tested in LDA for reactivity to donor HLA-DR peptides. Reactivity in $>50 \%$ of the samples obtained 6 mo after transplantation was considered indicative of persistent sensitization to donor alloantigens.

acquired recognition of new determinants, a process known as determinant spreading, is implicated in relapse and disease progression (40). Epitope spreading may also play a role in chronic allograft rejection by amplifying the alloimmune response. In previous studies, we showed that during primary rejection episodes, $\mathrm{T}$ cell reactivity in the peripheral blood, as well as in the graft, was directed against a single dominant alloepitope of one of the donor's HLA-DR antigens. Intermolecular spreading of $\mathrm{T}$ cell reactivity from one mismatched DR antigen to the other was observed in only four recipients, in conjunction with multiple early rejection episodes (31). These four recipients continued to display reactivity against both DR antigens of the donor at later times after transplantation.

LDA studies, performed after this initial 6-mo period, revealed intermolecular spreading in an additional 9 out of 27 patients with grafts mismatched by two DR alleles. Histologic evidence of late acute rejection grade $1 \mathrm{~B}$ to 3 was present in four recipients, yet absent in the remaining five recipients at the time when $T$ cell reactivity against the second HLA-DR antigen was observed. Fig. $3 A$ illustrates the phenomenon of intermolecular spreading in a patient with multiple rejection episodes who developed CAV. Overall, within the subset of

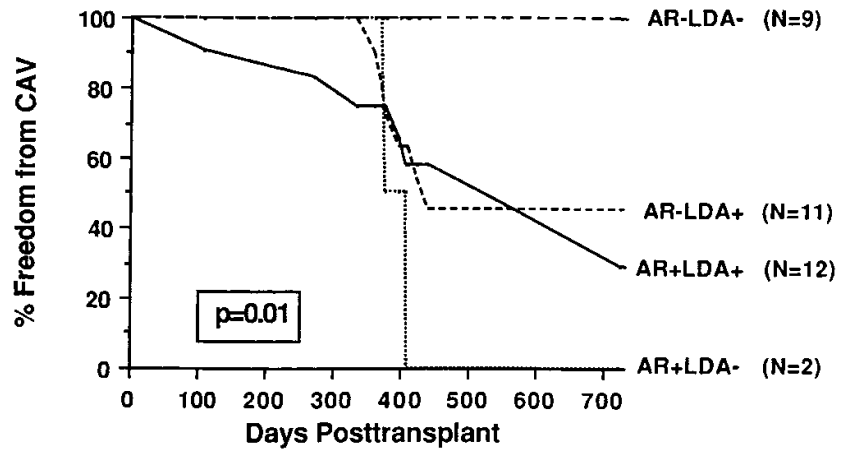

Figure 2. Relationship between late episodes of acute rejection, $\mathrm{T}$ cell reactivity to donor allopeptides, and CAV. Acute rejection episodes were monitored by EMB and considered to be present when graded histologically $1 \mathrm{~B}$ or higher. $\mathrm{T}$ cell reactivity to donor allopeptides was determined by LDA and considered positive if $>1.5 \times 10^{5}$ Th cells responding to donor HLA-DR peptides were detected. 

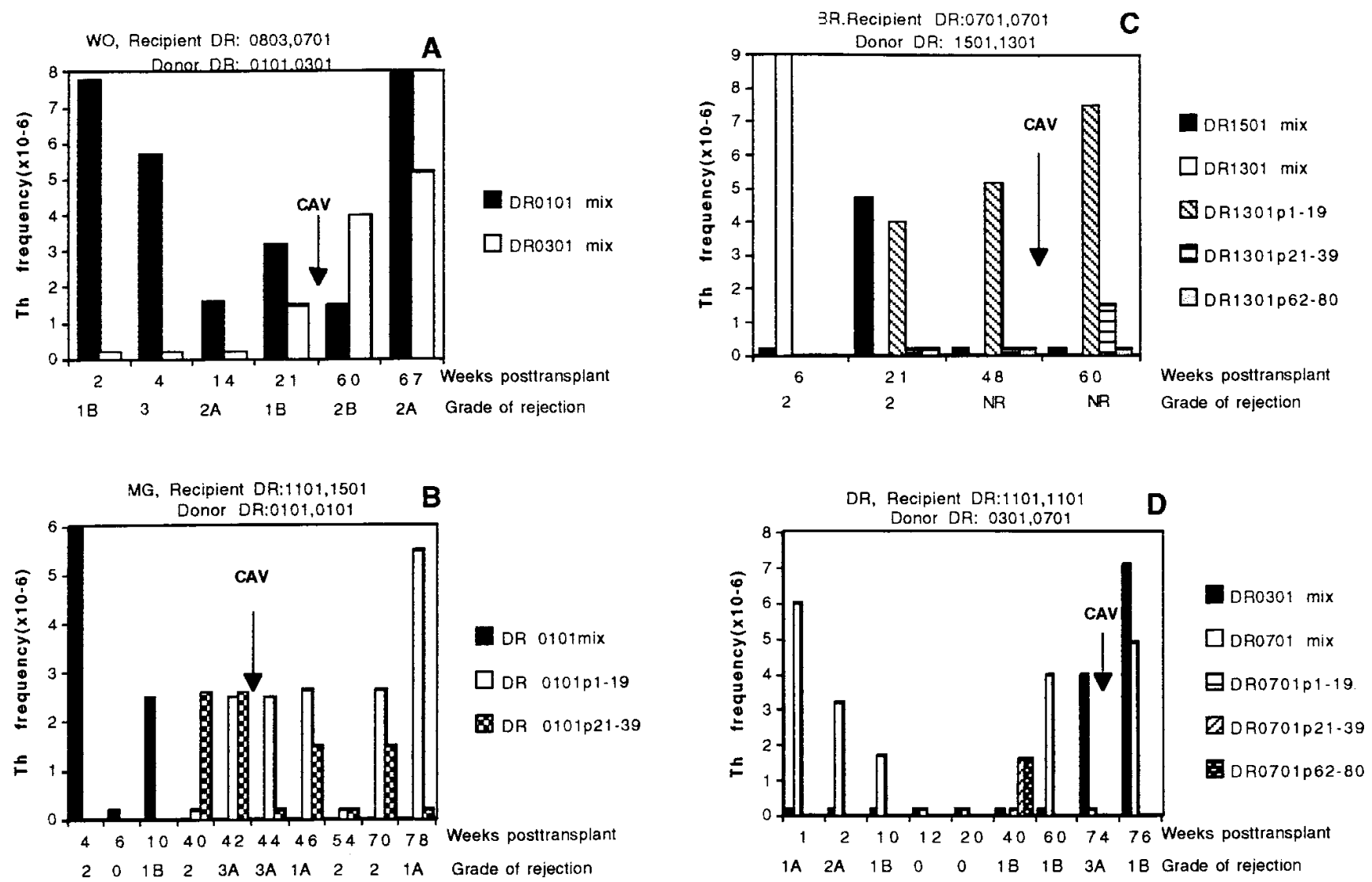

Figure 3. Inter- and intramolecular epitope spreading in patients with CAV. T cell reactivity against peptides corresponding to each of the mismatched HLA-DR antigens of the donor was tested serially in LDA to determine the specificity of the response. $(A)$ Intermolecular spreading of T cell reactivity from one mismatched HLA-DR (DR1) antigen to the other (DR3). (B) To establish whether intramolecular spreading has occurred, individual components of the mixture of peptides which was known to elicit stimulation were used instead of the mixture itself. Intramolecular spreading of T cell reactivity from an immunodominant (peptide 21-39) to a cryptic determinant (peptide 1-19) of the DR $\beta 1 * 0101$ antigen in a recipient carrying the DR $\beta 1 * 1101$ allele. $(C)$ Intermolecular spreading of T cell reactivity from DR13 to DR15 and intramolecular spreading from the immunodominant DR13 peptide 1-19 to the cryptic peptide 21-39. (D) Intramolecular spreading of T cell reactivity to two HLA-DR $\beta 1 * 0701$ peptides (peptide 1-19 and 21-39) and intermolecular spreading of the recipient's response from DR $\beta 1 * 0701$ to DR $\beta 1 * 0301$ alloantigen of the graft.

patients transplanted with a two-HLA-DR-mismatched graft $(n=27)$, the incidence of CAV in $<2$ yr was $62 \%(8 / 13)$ when intermolecular spreading occurred, compared with $44 \%(4 / 9)$ in recipients whose reactivity was confined to a single HLADR alloantigen, and $0 \%(0 / 5)$ in those who did not respond to donor allopeptides (Fig. 4). There was a significant association between intermolecular epitope spreading and the development of CAV $(P<0.02)$, suggesting that responses to secondary alloepitopes play a role in the pathology of chronic rejection. Hence, progression of chronic rejection may be due to the activation and recruitment of $T$ cells which recognize additional specificities.

To investigate the possibility that intramolecular epitope spreading also takes place, we mapped the epitopes recognized by allopeptide reactive $T$ cells at various times after transplantation. Analysis of the determinants recognized by patients' $\mathrm{T}$ cells during early rejection episodes showed that the response was directed against a sole immunodominant epitope of a mismatched HLA-DR antigen (31).

Studies performed on 12 patients with persistent allopep- tide reactivity 6 mo after transplantation, however, revealed intramolecular spreading of DR epitopes in 9 recipients (Table II). In these experiments, $\mathrm{T}$ cells obtained from sequential samples of blood were tested in LDA for reactivity against each individual peptide of the DR antigen recognized in a previous determination (performed with peptide mixtures). Reactivity to multiple peptides from the same DR antigen can be defined as intramolecular spreading if the peptides are presented by the same host restriction element. Alternatively, different peptides derived from the same alloantigen of the donor may be presented by the two HLA-DR antigens of an HLA-DR heterozygous recipient. To discriminate between intramolecular spreading and diversification of $\mathrm{MHC}$ restriction elements, $T$ cells from LDA studies were tested for allopeptide reactivity in the presence of APCs sharing with the recipient a single DR molecule.

Restriction experiments demonstrated that transplant patients who were HLA-DR heterozygous (BC, MG, CG) recognized different determinants of a mismatched HLA-DR antigen of the donor in the context of a single host HLA-DR 


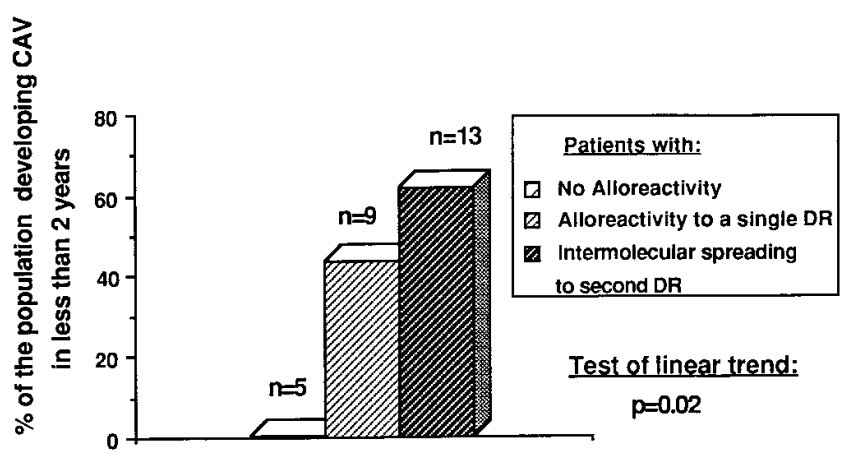

Figure 4. Relationship between CAV and intermolecular spreading of T cell epitopes. PBMCs from patients carrying a heart transplant mismatched by two HLA-DR antigens were tested for self-restricted reactivity against both DR alloantigens.

molecule (Table II). This biased recognition of donor DR presented by one, but not the other, host restriction element, is consistent with our previous findings (31). Dominant determinants are defined as those epitopes to which T cells initially respond when primed to native protein. Responses to other epitopes on that molecule which arrive later or upon hyperimmunization are termed cryptic (41). Immunodominant determinants were distinguished from cryptic epitopes based on the capacity of peptide-reactive $\mathrm{T}$ cells to recognize soluble protein of the corresponding HLA-DR antigen. The dominant epitope of the DR $\beta 1 * 0101$ molecule recognized by $\mathrm{T}$ cells in the context of the DR $\beta 1 * 1101$ antigen was shown previously to correspond to residues 21-39, while peptide 1-19 was found to correspond to a cryptic epitope. Peptide $62-80$ was not stimulatory (37).

In two patients (BC and $\mathrm{MG}$ ) who carried the $\mathrm{DR} \beta 1 * 1101$ antigen, the immunodominant epitope of the DR $\beta 1 * 0101$ molecule, peptide 21-39, was recognized during early rejection episodes occurring within the first 4 wk after transplantation. Intramolecular spreading was evidenced at later times after transplantation (70 and $42 \mathrm{wk}$, respectively) when the specificity of the $\mathrm{T}$ cell response shifted from the immunodominant peptide 21-39 to peptide 1-19 of the DR $\beta 1 * 0101$ molecule expressed by the graft (Table II). The response was directed either against both the dominant and cryptic epitope or only against one of these determinants at later times when the onset of CAV was diagnosed (Fig. $3 \mathrm{~B}$ ).

One of the recipients studied (BR) was a DR $31 * 0701$ homozygote transplanted with a graft carrying the DR $\beta 1 * 1501$, 1301 antigens. This patient's T cells reacted first against peptide 1-19 and later against peptide 21-39 of the DRß1*1301 antigen (Fig. $3 \mathrm{C}$ ). To identify the dominant epitope of the DR $\beta 1 * 1301$ molecule, we generated TCLs by in vitro immunization of PBMCs from a healthy DR $\beta 1 * 0701$ homozygote with the DR $\beta 1 * 1301$ peptides 1-19 and 21-39. Blastogenesis assays in which the resulting TCLs were stimulated with the corresponding peptide and with soluble DR $\beta 1 * 1301$ protein showed that the TCL primed with peptide 1-19 recognized the soluble DR $\beta 1 * 1301$ protein, while the TCL specific for peptide 21-39 did not. This indicates that peptide 1-19 is dominant, while peptide 21-39 is cryptic when presented by DR7-positive APCs (Fig. 5, $A$ and $B$ ). This information pertaining to patient BR (Table II and Fig. $3 C$ ) indicates that the response spread from an immunodominant to a cryptic epitope with progression of the rejection process. Hence, knowledge of the hierar-

Table II. Intramolecular Spreading of T Cell Epitopes

\begin{tabular}{|c|c|c|c|c|c|c|c|}
\hline \multirow{2}{*}{$\begin{array}{l}\text { Patient } \\
\text { initial }\end{array}$} & \multicolumn{2}{|c|}{ HLA-DR genotype } & \multirow{2}{*}{$\begin{array}{c}\text { Restriction } \\
\text { element }\end{array}$} & \multirow{2}{*}{$\begin{array}{c}\text { T cell } \\
\text { determinant }\end{array}$} & \multirow{2}{*}{$\begin{array}{l}\text { Time of epitope } \\
\text { spreading } \\
\text { (weeks after } \\
\text { transplantation) }\end{array}$} & \multirow{2}{*}{$\begin{array}{c}\text { Rejection } \\
\text { episodes } \\
\text { (weeks after } \\
\text { transplantation) }\end{array}$} & \multirow{2}{*}{$\begin{array}{c}\text { CAV status } \\
(+/-)\end{array}$} \\
\hline & Recipient & Donor & & & & & \\
\hline $\mathrm{BC}$ & 0701,1101 & 0101,0401 & 1101 & $\begin{array}{l}0101 \text { pp } 21-39 * \\
0101 \text { pp } 1-19\end{array}$ & 70 & $1,2,10,13,70$ & + \\
\hline BR & 0701,0701 & 1501,1301 & 0701 & $\begin{array}{l}1301 \text { pp } 1-19 * \\
1301 \text { pp } 21-39\end{array}$ & 50 & $6,16,21$ & + \\
\hline MG & 1501,1101 & 0101,0101 & 1101 & $\begin{array}{l}0101 \text { pp } 21-39 * \\
0101 \text { pp } 1-19\end{array}$ & 42 & $4,10,40,54,70$ & + \\
\hline MH & 1502,1502 & 1501,1104 & 1502 & $\begin{array}{l}1104 \text { pp } 21-39 * \\
1104 \text { pp } 1-19 \\
1104 \text { pp } 62-80\end{array}$ & 42 & $1,4,40,48,54,73$ & - \\
\hline $\mathrm{BJ}$ & 1101,1101 & 1501,1601 & 1101 & $\begin{array}{l}1501 \text { pp } 1-19 \\
1501 \text { pp } 21-39\end{array}$ & 52 & $2,24,36$ & - \\
\hline $\mathrm{BA}$ & 1404,0401 & 1301,1305 & NT & $\begin{array}{l}1305 \text { pp } 1-19 \\
1305 \text { pp } 21-39 \\
1305 \text { pp } 62-80\end{array}$ & 67 & 2,5 & - \\
\hline $\mathrm{CG}$ & 1501,0701 & 1201,1501 & 1501 & $\begin{array}{l}1201 \text { pp } 1-19 \\
1201 \text { pp } 21-39\end{array}$ & 40 & $1,7,39$ & - \\
\hline DR & 1101,1101 & 0701,0301 & 1101 & $\begin{array}{l}0701 \text { pp } 21-39 \\
0701 \text { pp 62-80 }\end{array}$ & 40 & $2,10,40,60,74$ & + \\
\hline NR & 0301,1201 & 1104,1305 & NT & $\begin{array}{l}1104 \text { pp } 1-19 \\
1104 \text { pp 21-39 }\end{array}$ & 63 & $1,15,41$ & - \\
\hline
\end{tabular}

*Immunodominant. 

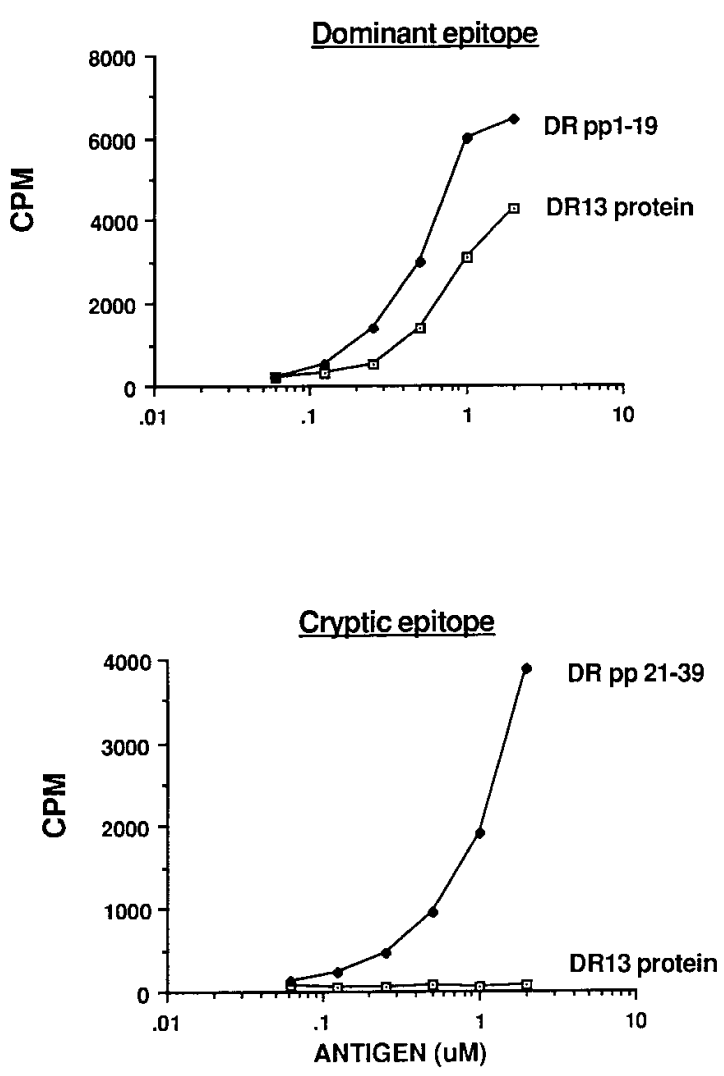

Figure 5. Identification of the dominant T cell epitope of the HLADR $\beta 1 * 1301$ molecule presented by the DR $\beta 1 * 0701$ antigen. PBMCs from a DR $\beta 1 * 0701$ homozygous individual were immunized in vitro with synthetic peptides corresponding to residues 1-19 $(A)$ and 21-39 $(B)$ of the DR $\beta 1 * 1301$ antigen. The resulting TCLs were tested in blastogenesis assay for reactivity against the immunizing peptide and soluble DR 1301 protein obtained by immunoaffinity purification from a B cell line.

chy of determinants recognized in the context of distinct MHC molecules permitted us to establish the order in which the response of four heart allograft recipients spread to a new determinant of the same HLA-DR molecule expressed by the graft (patient BC, BR, MG, and MH from Table II). In each of these four cases, the immunodominant determinant was recognized before the response shifted to a cryptic epitope, suggesting that spreading followed an ordered pattern. The immunodominant determinant recognized by the remaining five patients (BJ, BA, CG, DR, and NR) has not been mapped yet. Also, the sequence in which the peptides were recognized by $\mathrm{T}$ cells from these five recipients could not be established since reactivity to two or three peptides was already present at the time when intramolecular spreading was first tested.

The mean number of rejection episodes detected in patients displaying intramolecular spreading of $\mathrm{T}$ cell epitopes was three (range of two to six). Two of the three patients who showed no intramolecular spreading had a single rejection, and one had two rejection episodes. This observation together with the finding that intramolecular spreading occurred relatively late (i.e., after 6 mo after transplantation) suggest that $\mathrm{T}$ cells recognizing new determinants are activated after extensive allograft damage has occurred. Both intra- and intermo- lecular spreading was observed in some patients developing CAV (Fig. 3, $C$ and $D$ ), supporting the hypothesis that chronic rejection may be due to the activation and recruitment of alloreactive $\mathrm{T}$ cells with novel specificities.

\section{Discussion}

Indirect allorecognition of donor HLA alloantigens may be crucial to the development of CAV by providing help for alloantibody production and by promoting lymphokine secretion required for delayed-type hypersensitivity and cytotoxic T cell activity $(31,32)$. Persistent allopeptide reactivity and epitope spreading may result in chronic $\mathrm{CD} 4^{+} \mathrm{T}$ cell-mediated injury to the graft. By analogy with the model proposed in autoimmune diseases, it is possible that $\mathrm{T}$ cells with direct recognition ability trigger the inflammatory cascade in the graft resulting in tissue damage. HLA antigens shed from the injured graft may then be processed by macrophages and antigen specific B cells, and presented in a self-restricted manner to allopeptide specific $\mathrm{T}$ cells. Upon activation, such $\mathrm{T}$ cells may fuel the inflammatory response (42). Antigen specific B cells, which are significantly more efficient than other APCs, may be responsible for the diversification of the immune response $(42,43)$.

Evidence supporting this hypothesis derives from previous studies in which chronic rejection was shown to be associated with the release of soluble HLA alloantigens and the development of anti-HLA antibodies reflecting the activation of alloantigen specific B cells in the lymphoid system $(6,44)$. Processing of antigen-antibody complexes may result in the diversification of $\mathrm{T}$ cell epitopes. Furthermore, this model is consistent with our previous and present findings that during early primary rejection episodes, T cell alloreactivity is directed against a sole immunodominant epitope of donor HLA-DR molecules; at later times, the response diversifies to include multiple determinants of the same or other antigens. Although numerous experimental studies have documented the contribution of indirect allorecognition to acute rejection (21-23, 32, 45-47), no animal model has been developed so far to explore the self-restricted $\mathrm{T}$ cell alloresponse in chronic rejection.

In this report, we have studied the relationship between persistent alloreactivity, epitope spreading, and development of CAV. Using synthetic peptides corresponding to the hypervariable region of 32 HLA-DR alleles, we have investigated the specificity of self-restricted $\mathrm{T}$ cell alloresponses to the donor after the first 6 mo after transplantation, when chronic rejection may occur. 14 heart allograft recipients from this population of 34 patients developed CAV within $<2 \mathrm{yr}$. A strong correlation between donor specific allopeptide reactivity and both acute (31) and chronic rejection was found. The incidence of CAV was significantly higher in patients who continued to respond against donor HLA-DR peptides late after transplantation, even when there was no evidence of acute rejection, compared with patients without persistent alloreactivity. In only two cases did chronic rejection occur in the absence of late allopeptide reactivity. However, both of these patients displayed early reactivity to donor allopeptides. It is possible that the lesions resulting in chronic rejection already occurred during the first $6 \mathrm{mo}$ and progressed thereafter in spite of suppression of T cell reactivity to donor allopeptides by some kind of a regulatory mechanism. Nonimmunologic mechanisms such as the secretion of growth factors by the parenchymal and endo- 
thelial cells in the allograft, in response to early immune injury, may lead to proliferation of smooth muscle cells and influx of myocytes into the intima producing allograft inflammation and arteriosclerosis $(48,49)$. Therefore, it is possible that chronic rejection may also be caused by injury occurring during early acute rejection episodes.

The correlation between allopeptide reactivity and development of atherosclerosis supports the hypothesis that CAV results from continual injury of the cells of the vascular endothelium by lymphokines produced by allopeptide-reactive $\mathrm{T}$ cells. The chronicity of the $\mathrm{T}$ cell response may be due to incomplete immunosuppression or to a breakdown in immunoregulatory suppressive mechanism(s). Monitoring of donor specific allopeptide reactivity by LDA provides a useful strategy for identifying patients at risk for the development of CAV.

Similar to the situation encountered during the progression of autoimmune diseases, the development of CAV was associated with epitope spreading $(35,36,40-42)$. Although the mechanism of epitope spreading is not completely understood, it has been postulated that the choice of which $\mathrm{T}$ cell epitopes will become dominant should depend on the antigen processing pattern occurring in different APCs. This is, in turn, related to the efficiency of internalization and denaturation of the antigen (32, 50-52). APCs of different cell lineages in different organs can process differently $(32,41,53)$. It has been suggested that "novel" (cryptic) peptides are generated as a result of either of the following mechanisms: (a) $\mathrm{Th}_{1}$-cytokineinduced alteration of endosomal enzymes with consequent changes in the cleavage of proteins during antigen processing; (b) the recruitment of nonprofessional APCs, such as macrophages, epithelial, and endothelial cells; (c) upregulation of MHC class II expression and induction of cellular proteases by IFN- $\gamma$; and $(d)$ inherent differences in the processing machinery displayed by different APCs such as B cells, other splenic APCs, and peritoneal macrophages (54).

Furthermore, recent studies suggest that an allelic polymorphism in antigen processing cofactor genes contributes to the selection of MHC peptides available for presentation by MHC class II molecules, playing an important role in direct and indirect $\mathrm{T}$ cell recognition of alloantigens (21).

In this study, we assessed the specificity of indirect recognition by identifying the immunogenic $\mathrm{T}$ cell determinants of the hypervariable region of the first domain of donor HLA-DR $\beta 1$ molecules recognized by the recipient at various times after transplantation. A hierarchy of determinants was found since mapping of alloreactivity to HLA-DR antigens revealed intramolecular spreading of the response from the immunodominant determinant to additional determinants which behaved cryptically at earlier times after heart transplantation. Hence, the order in which intramolecular spreading occurred followed the traditional immunodominant-nondominant hierarchy (21, 41). Furthermore, recipients carrying a graft mismatched by two HLA-DR antigens showed intermolecular spreading of reactivity from one HLA-DR antigen of the donor to the other. The strong association between epitope spreading and development of CAV suggests that the inflammatory cytokines associated with recurrent acute episodes and chronic rejection are due, at least in part, to spreading of $\mathrm{T}$ cell reactivity to new allodeterminants.

Since the frequency of allopeptide specific T cells varied at different points in time, it is possible that this response is subjected to immunoregulatory mechanisms and/or that alloreac- tive $\mathrm{T}$ cells specific for the immunodominant determinant may be suppressed upon exposure to high concentrations of allopeptide-self MHC complexes (37). This may explain the weakening of the response to one determinant and subsequent reactivity to spreading determinants. If so, the development of chronic rejection may require the persistence of a certain threshold of inflammatory responses which are perpetuated through a determinant-spreading cascade. Risk factors such as acute rejection episodes, or infections, may provide further opportunities for damage to the allograft vascular endothelia, shedding of upregulated HLA-DR antigens, and immunostimulation.

We have demonstrated recently that direct recognition of donor HLA-DR antigens can also be measured using the activation markers CD69 and LDA1 (CD103) for flow cytometric determination of recipient $\mathrm{T}$ cells which become activated after stimulation with donor B cells (55). Although this method provides a rapid tool for detecting antidonor-reactive $\mathrm{T}$ cells, some early acute rejection episodes are "missed" unless T cell reactivity to donor allopeptide is also tested. This suggests that allopeptide reactivity is a more sensitive tool for detecting host immune activation against donor HLA antigens (55).

The dynamics of $\mathrm{T}$ cell responses to allopeptides provides a rationale for therapeutic intervention at different times after transplantation. While immunodominant peptides may be efficient in suppressing alloreactivity if administered before or at the time of primary rejection episode, patients with multiple rejection may benefit from treatment with peptides corresponding to both dominant and nondominant determinants. Knowledge of the dynamics of indirect alloreactivity may serve both to target and monitor the effectiveness of therapeutic strategies.

\section{Acknowledgments}

The authors acknowledge Nicole Foca-Rodi and Alexandra Vasilescu for their valuable editorial assistance.

This work was supported in part by National Institutes of Health grant RO1-A12521 0-10.

\section{References}

1. Miller, L.W., T. Donahue, T. Wolford, and J. Drury. 1996. In The Transplantation and Replacement of Thoracic Organs. D.K. Cooper, L.W. Miller, and G.A. Patterson, editors. Kluwer Academic Publishers, Boston. 335-350.

2. Stinson, E.B., R. Payne, R.B. Griepp, and E. Dong, Jr. 1971. Correlation of histocompatibility matching with graft rejection and survival after cardiac transplantation in man. Lancet. 2:459-461.

3. Frist, W., P. Oyer, J. Baldwin, E.B. Stinson, and N.E. Shumway. 1987. HLA compatibility and cardiac transplant recipient survival. Ann. Thorac. Surg. 44:242-246.

4. Smith, J., M.L. Rose, A. Pomerance, M. Burke, and M.H. Yacoub. 1995. Reduction of cellular rejection and increase in long-term survival after heart transplantation, after HLA-DR matching. Lancet. 346:1318-1322.

5. Costanzo-Nordin, M. 1992. Cardiac allograft vasculopathy: relationship with acute cellular rejection and histocompatibility. J. Heart Lung Transplant. 11:S90-S103.

6. Suciu-Foca, N., E. Reed, C. Marboe, Y.-P. Xi, S.-Y. Kai, E. Ho, E. Rose, K. Reemtsma, and D.W. King. 1991. Role of anti-HLA antibodies in heart transplantation. Transplantation. 51:716-724.

7. Hess, M., A. Hastillo, T. Mohanakumar, J.J. Cowley, G. Vetrovac, S. Szentpetery, T.C. Wolfgang, and R.R. Lower. 1983. Accelerated atherosclerosis in cardiac transplantation: role of cytotoxic B cell antibodies and hyperlipidemia. Circulation. 68:94-101.

8. Rose, E., P. Pepino, M. Barr, C. Smith, A. Ratner, E. Ho, and C. Berger. 1992. Relation of HLA antibodies and graft atherosclerosis in human cardiac allograft recipients. J. Heart Lung Transplant. 11:Sl20-S123.

9. Rose, E., C. Smith, G. Petrossian, M.L. Barr, and K. Reemtsma. 1989 
Humoral immune responses after cardiac transplantation: correlation with fatal rejection and graft atherosclerosis. Surgery. 106:203-208.

10. Fenoglio, J., E. Ho, E. Reed, E. Rose, C. Smith, K. Reemtsma, C. Marboe, and N. Suciu-Foca. 1989. Anti-HLA antibodies and heart allograft survival. Transplant. Proc. 21:807-809.

11. Petrossian, G., A.B. Nichols, C.C. Marboe, R. Sciacca, E.A. Rose, C.R. Smith, P.J. Cannon, K. Reemtsma, and E.R. Powers. 1989. Relation between survival and development of coronary artery disease and anti-HLA antibodies after cardiac transplantation. Circulation. 80:122-125.

12. Hayry, P. 1996. Pathophysiology of chronic rejection. Transplant. Proc. 28:7-10.

13. Hayry, P., S. Mennander, J. Yilmarz, J. Ustinov, A. Raisanen, A. Miettinen, I. Lautenschlager, K. Lemstrom, C.A. Bruggerman, and T. Paavonen. 1992. Towards understanding the pathophysiology of chronic rejection. Clin. Invest. 70:780-790.

14. Demetris, A.J., T. Zerbe, and B. Banner. 1989. Morphology of solid organ allografts arteriopathy. Identification of proliferating intimal cell populations. Transplant. Proc. 21:3667-3669.

15. Thyberg, J., U. Hedin, M. Sjolund, L. Palmberg, and B.A. Bottger. 1989. Regulation of differentiated properties and proliferation of arterial smooth muscle cells. Arteriosclerosis. 10:966-990.

16. Foegh, M.L. 1988. Eicosanoids and platelet activating factor. Mechanism in organ rejection. Transplant. Proc. 20:1260-1263.

17. Gordon, D. 1992. Growth factors and cell proliferation in human transplant arteriosclerosis. J. Heart Lung Transplant. 11:S7.

18. Allen, M., T. McDonald, T. Carlos, V. Himes, D. Fishbein, S. Aziz, and D. Gordon. 1992. Endothelial adhesion molecules in heart transplantation. J. Heart Lung Transplant. 11:S8-S13.

19. Libby, P. 1990. Inflammatory and immune mechanisms in atherogenesis. Atherosclerosis Rev. 21:79-89.

20. Hayry, P., T. Paavonen, A. Mennander, J. Ustinov, S.A. Raisanen, and K. Semstrom. 1993. Pathophysiology of allograft arteriosclerosis. Transplant. Proc. 25:2070-2071.

21. Benichou, G., and E.V. Fedoseyeva. 1996. The contribution of peptides to T cell allorecognition and allograft rejection. Intern. Rev. Immunol. 13:231-243. 22. Bradley, J.A. 1996. Indirect T cell recognition in allograft rejection. Intern. Rev. Immunol. 13:245-255.

23. Sayegh, M.H., and C.B. Carpenter. 1996. Role of indirect allorecognition in allograft rejection. Intern. Rev. Immunol. 13:221-229.

24. Colovai, A.I., E. Renna Molajoni, R. Cortesini, and N. Suciu-Foca. 1996. New approaches to specific immunomodulation in transplantation. Intern. Rev. Immunol. 13:161-172.

25. Lechler, R.I., G. Lombardi, J.R. Batchelor, N. Reinsmoen, and F.H. Bach. 1990. The molecular basis of alloreactivity. Immunol. Today. 11:83-88

26. Lechler, R.I., T. Heaton, L. Barber, V. Ball, J.R. Batchelor, and G. Lombardi. 1992. Molecular mimicry by major histocompatibility complex molecules and peptides accounts for some alloresponses. Immunol. Lett. 34:63-70.

27. Mason, P.D., C.M. Robinson, and R.I. Lechler. 1996. Detection of donor-specific hyporesponsiveness following late failure of human renal allografts. Kidney Int. 50:1019-1025.

28. Quill, H., and R.H. Schwartz. 1987. Stimulation of normal inducer T cell clones with antigen presented by purified la molecules in planar lipid membranes: specific induction of a long-lived state of proliferative non-responsiveness. J. Immunol. 138:3704-3712.

29. Jenkins, M.K., D.M. Pardoll, J. Mizuguchi, T.M. Chused, and R.H. Schwartz. 1987. Molecular events in the induction of nonresponsive state in interleukin 2 producing helper T-lymphocyte clones. Proc. Natl. Acad. Sci. USA. 84:5409-5413.

30. Braun, M.Y., A. McCormack, G. Webb, and J.R. Batchelor. 1993. Evidence for clonal anergy as a mechanism for the maintenance of transplantation tolerance. Eur. J. Immunol. 23:1462-1468.

31. Liu, Z., A.I. Colovai, S. Tugulea, E.F. Reed, P.E. Fisher, D. Mancini, E.A. Rose, R. Cortesini, R.E. Michler, and N. Suciu-Foca. 1996. Indirect recognition of donor HLA-DR peptides in organ allograft rejection. J. Clin. Invest. 98:1150-1157.

32. Benichou, G., E. Fedoseyeva, P.V. Lehmann, C.A. Olson, H.M. Geysen, M. McMillan, and E.E. Sercarz. 1994. Limited T cell response to donor MHC peptides during allograft rejection. J. Immunol. 153:938-945.

33. Sercarz, E.E., and U. Krzych. 1991. The distinctive specificity of antigen-specific suppressor T cells. Immunol. Today. 12:111-118.

34. Clayton, J.P., G. Gammon, D.G. Ando, D.H. Kono, L. Hood, and E.E. Sercarz. 1989. Peptide-specific prevention of experimental allergic encephalo- myelitis: neonatal tolerance induced to the dominant $\mathrm{T}$ cell determinant of myelin basic protein. J. Exp. Med. 169:1681-1691.

35. McRae, B.L., C.L. Vanderlugt, M.C. Dal Canto, and S.D. Miller. 1995. Functional evidence for epitope spreading in the relapsing pathology of experimental autoimmune encephalomyelitis. J. Exp. Med. 182:75-85.

36. Lehmann, P.V., E.E. Sercarz, T. Forsthuber, C.M. Dayan, and G. Gammon. 1993. Determinant spreading and the dynamics of the autoimmmune $T$ cell repertoire. Clin. Immunol. Immunopathol. 14:203-208.

37. Liu, Z., P. Harris, A.I. Colovai, E.F. Reed, A. Maffei, and N. SuciuFoca. 1996. Indirect recognition of donor MHC class II antigens in human transplantation. Clin. Immunol. Immunopathol. 78:228-235.

38. Zhang, J., S. Markovic-Plese, B. Lacet, J. Rays, H.L. Weiner, and D.A. Hafler. 1994. Increased frequency of interleukin 2-responsive T cells specific for myelin basic protein and proteolipid protein in peripheral blood and cerebrospinal fluid of patients with multiple sclerosis. J. Exp. Med. 179:973-984.

39. Harris, P.E., Z. Liu, and N. Suciu-Foca. 1992. MHC class II binding of peptides derived from HLA-DR1. J. Immunol. 148:2169-2174.

40. Yu, M., J.M. Johnson, and V.K. Tuohy. 1996. A predictable sequential determinant spreading cascade invariably accompanies progression of experimental autoimmune encephalomyelitis: a basis for peptide-specific therapy after onset of clinical disease. J. Exp. Med. 183:1777-1788.

41. Sercarz, E.E., P.V. Lehmann, A. Ametani, G. Benichou, A. Miller, and K. Moudgil. 1993. Dominance and crypticity of T cell antigenic determinants. Annu. Rev. Immunol. 11:729-766.

42. Vanderlugt, C.J., and S.D. Miller. 1996. Epitope spreading. Curr. Opin. Immunol. 8:831-836.

43. Mamula, M.J., and C.A. Janeway, Jr. 1993. Do B cells drive the diversification of immune responses? Immunol. Today. 14:151-154.

44. Reed, E.F., B. Hong, E. Ho, P.E. Harris, J. Weinberger, and N. SuciuFoca. 1996. Monitoring of soluble HLA alloantigens and anti-HLA antibodies identifies heart allograft recipients at risk of transplant associated coronary artery disease. Transplantation. 61:556-572.

45. Sayegh, M.H., B. Watschinger, and C.B. Carpenter. 1994. Mechanisms of allorecognition. Recognition by in vivo primed $\mathrm{T}$ cells of specific major histocompatibility complex polymorphisms presented as peptides by responders presenting cells. Transplantation. 57:572-576.

46. Gallon, L., B. Watschinger, B. Murphy, E. Akalin, M.H. Sayegh, and C.B. Carpenter. 1995. The indirect pathway of allorecognition. The occurrence of self-restricted $\mathrm{T}$ cells recognition of allo $\mathrm{MHC}$ peptides early in acute renal allograph rejection and its inhibition by convention immunosuppression. Transplantation. 59:612.

47. Sayegh, M.H., and B. Carpenter. 1996. Role of indirect allorecognition in allograft rejection. Int. Rev. Immunol. 13:221-229.

48. Hosenpud, J.D., T.E. Morris, G.D. Shipley, K.A. Mauck, and C.R. Wagner. 1996. Cardiac allograft vasculopathy. Preferential regulation of endothelia cell-derived mesenchymal growth factors in response to a donor-specific cellmediated allogeneic response. Transplantation. 61:939-948.

49. Wagner, C.R., T.E. Morris, G.D. Shipley, and J.D. Hosenpud. 1993 Regulation of human aortic endothelial cell-derived mesenchymal growth factors by allogeneic lymphocytes in vitro. A potential mechanism for cardiac allograft vasculopathy. J. Clin. Invest. 92:1269-1277.

50. Lanzavecchia, A. 1985. Antigen-specific interactions between T and B cells. Nature. 314:537-539.

51. Casten, L.A., and S.K. Pierce. 1988. Receptor-mediated B cell antigen processing: increased antigenicity of a globular protein covalently coupled to antibodies specific for B cell and cell clones stimulated by macrophages exposed to antigen complexed with monoclonal antibodies: a possible influence of paratope specificity on the mode of antigen processing. J. Immunol. 140:404-410.

52. Yoshikawa, M., M. Watanabe, and N. Hozumi. 1987. Analysis of proteolytic processing during specific antigen presentation. Cell. Immunol. 110: 431-435.

53. Vidard, L., K.R. Rock, and B. Benacerraf. 1992. Heterogeneity in antigen processing by different types of antigen-presenting cells. J. Immunol. 149: 1905-1911.

54. Elson, C.J., R.N. Barker, S.J. Thompson, and N.A. Williams. 1995. Immunologically ignorant autoreactive T cells, epitope spreading and repertoire limitation. Immunol. Today. 16:71-76.

55. Tugulea, S., R. Ciubotariu, A.I. Colovai, Z. Liu, S. Itescu, L.L. Schulman, P.E. Fisher, M.A. Hardy, E.A. Rose, R.E. Michler, R. Cortesini, and N. Suciu-Foca. 1997. New strategies for early diagnosis of heart allograft rejection. Transplantation. 64:842-847. 